\title{
Mobile Receiver-Assisted Localization Based on Selective Coordinates in Approach to Estimating Proximity for Wireless Sensor Networks
}

\author{
Zulfazli Hussin \\ Graduate School of Applied Informatics \\ University of Hyogo \\ Kobe, Hyogo, Japan 650-0047 \\ Email: zulfazlihussin AT gmail.com
}

\author{
Yukikazu Nakamoto \\ Graduate School of Applied Informatics \\ University of Hyogo \\ Kobe, Hyogo, Japan 650-0047 \\ Email: nakamoto AT ai.u-hyogo.ac.jp
}

\begin{abstract}
Received signal strength (RSS)-based mobile localization has become popular due to its inexpensive localization solutions in large areas. Compared to various physical properties of radio signals, RSS is an attractive approach to localization because it can easily be obtained through existing wireless devices without any additional hardware. Although RSS is not considered to be a good choice for estimating physical distances, it provides some useful distance related information in adding and indicating connectivity information in neighboring nodes. RSS-based localization is generally divided into range-based and rangefree. Range-based localization can achieve excellent accuracy but is too costly to apply to large-scale networks. Methods of range-free localization are regarded as cost-effective solutions for localization in sensor networks. However, the localizations are subject to the effect of radio patterns that affect variations in the radial distance estimates between nodes. It is a challenging task to select an efficient RSS value that can provide small variations in the radial distance in wireless environments. We propose a method of Mobile Localization using the Proximities of Selective coordinates (MoLPS) to localize target nodes by using information on proximities between target nodes and mobile receivers as a metric to estimate the location of target nodes. We ran a simulation experiment to assess the performance of MoLPS with 100 target nodes that were randomly deployed along a sensory field boundary. We found from the results of the simulation experiment that localization error had been reduced to below $2 \mathrm{~m}$ in more than $80 \%$ of the target nodes.
\end{abstract}

Keywords-Localization, proximity estimation, genetic algorithm, wireless sensor networks, received signal strength.

\section{INTRODUCTION}

Wireless sensor networks (WSNs) [1] are composed of many sensor nodes that have sensing and computational and wireless communication capabilities. Although WSNs have demonstrated their importance and capabilities in emergency applications, if the positions of sensor nodes are known, the use of these applications could be even more effective.

Localization is fundamentally a serious problem that deals with how to use information from sensor nodes to determine position coordinates. Locating an item is a critical process at distribution centers since poor performance results in unsatisfactory customer services (long processing and lagged delivery) and high costs. Suppose that a sensor node is attached to an item at a distribution center. Although placing an item at a fixed location makes it easier to locate it, it is not always the most space-efficient method of storage for products that are less predictable due to uncertain demand [2]. In contrast, random-location storage uses less storage space even though it requires the use of a locator to identify the locations of items. A straightforward solution would be to equip all sensor nodes with GPS receivers that could provide them with the exact locations of items. However, this is not a cost-effective solution and it has limited applications because GPS only works in open areas with no obstructions to satellite signals.

Receiver-assisted localization has attracted a great deal of attention in estimating the positions of items that are equipped with sensor nodes. Receivers detect sensor nodes by using the radio signals received from them. There are generally two types of deployments used to detect sensor nodes. The first is to fix several receivers that cover particular regions [3]. Thus, the numbers of receivers and their distributions have a direct impact on the accuracy of localization. A large number of distributed receivers will lead to improved accuracy. However, costs will be high if they are applied to large areas. The second method is to use mobile receivers to sense locations. Since mobile receivers are portable and easy to use, they are suitable for location sensing in large areas (e.g. distribution centers).

On this basis, we reassess existing localization scheme and exlore the possibility of using selected coordinates of mobile receivers. We evaluated the concentration of center coordinates, which are computed from the selected coordinates of mobile receivers, to estimate the position of a target node without deploying fixed receivers or fixed anchors. We called the coordinates of the mobile receivers footprints. We divided the footprints into multiple sets in which each set represented the footprints that received signals in the given range of path loss values for each set. Path loss describes a signal's energy loss that varies continuously as it travels to a receiver [4]. Instead of selecting all the footprints to compute the average for each set, we selected footprints that had fewer variations in the radial distance to the true target node in each set. The center coordinates of selected footprints were individually computed and the concentration of center coordinates of selected footprints were evaluated to estimate the true position of the target node.

The rest of the paper is organized as follows: The motiva- 

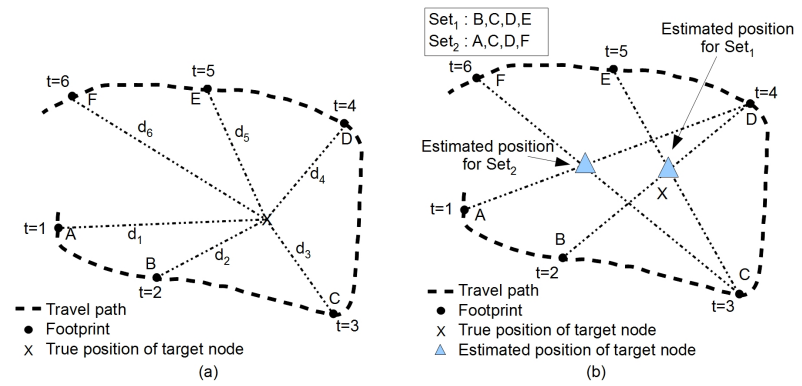

Fig. 1: (a) Variations in distance using centroid and (b) comparison of localization accuracy with two different selection of footprints

tion for our research is presented in Section 2. We investigated state-of-the-art range-based and range-free localization techniques, which are described in Section 3. Section 4 describes the problem of how to localize target nodes by using our proposed algorithm. Section 5 describes our overall algorithm. Our evaluation of performance is described in Section 6. and are followed by closing remarks in Section 7.

\section{Motivation}

Mobile receivers are used in mobile localization to measure the physical properties of collected radio signals from target nodes. Localization based on received signal strength (RSS) has become popular because it is an inexpensive solution to the problem of localizing target nodes. Compared to various physical properties of radio signals, such as Time of Arrival (ToA) [5], Time Difference of Arrival (TDoA) [6] or Angle of Arrival (AoA) [7], RSS is an attractive approach to localization because it can easily be obtained through existing wireless devices without the need for any additional hardware. The major challenge to accurate RSS-based positioning results from the variations in RSS that change over time and space due to dynamic and unpredictable signal propagation. Although RSS is not considered to be a good choice for estimating physical distances in many scenarios that involve unknown radio path loss factors, hardware discrepancies, and antenna orientation [8], [9], it provides useful information that is distance related in addition to indicating connectivity information between neighboring nodes. Many techniques of RSS-based localization have been proposed in the past two decades. They generally fall in two categories of range-based and range-free.

RSS readings are used in range-based localization techniques [8], [9] to directly estimate the physical location of target nodes. However, RSS measurements are easily corrupted by surrounding environments. Moreover, techniques of range-based localization require expensive and power-intensive measuring devices or synchronization that may incur cost and energy problems. In contrast, range-free approaches have been proposed as an alternative to pursue cost and energy effectiveness in WSNs [10], [11]. Range-free approaches are typically used for connectivity between nodes as a metric to estimate the position of target nodes without computing the actual distance between nodes.

The accuracy of localization in range-free approaches is subject to the effect of radio patterns that affect variations
TABLE I: Set of footprints.

\begin{tabular}{|cc|}
\hline Set, $q$ & Path Loss [dB], $P L$ \\
\hline 1 & $41.5<P L \leq 42.5$ \\
\hline 2 & $42.5<P L \leq 43.5$ \\
\hline 3 & $43.5<P L \leq 44.5$ \\
\hline 4 & $44.5<P L \leq 45.5$ \\
\hline 5 & $45.5<P L \leq 46.5$ \\
\hline 6 & $46.5<P L \leq 47.5$ \\
\hline 7 & $47.5<P L \leq 48.5$ \\
\hline 8 & $48.5<P L \leq 49.5$ \\
\hline 9 & $49.5<P L \leq 50.5$ \\
\hline 10 & $50.5<P L \leq 51.5$ \\
\hline
\end{tabular}

in estimates of the radial distance between nodes. Many of these techniques use an average of all anchor positions in their communication range [10] or in the same hop-count values [11] to localize the target nodes, which results in variations in the radial distance being underestimated thereby causing large localization errors. Localization errors vary between estimates of target nodes caused by variations in the radial distance that result from target nodes that have not been uniformly deployed. It is a challenging task to select efficient RSS values that can provide small variations in the radial distance from accumulated RSS values in wireless environments where complex and dynamic RSS values can affect the estimates of radial distances.

We propose a method of Mobile Localization using Proximities of Selective coordinates (MoLPS) to localize target sensor nodes by using the connectivity between them and mobile receivers as a metric to estimate their locations to solve these stated challenges. Locations are estimated from the coordinates of footprints where the signals are collected from target sensor nodes. MoLPS assume the presence of a tentative coordinate that is arbitrarily located at a known location in the field in which it is deployed. The distance between a tentative coordinate and center coordinates of selected footprints are iteratively compared to select effective footprints to localize target nodes. We used a genetic algorithm (GA) to search the best selection of footprints that had fewer variations in the radial distance from other selected footprints to the tentative coordinates. We iteratively improved the positions of the tentative coordinate by evaluating the concentration of center coordinates of selected footprints in the vicinity of the tentative coordinate.

\section{RELATED WORKS}

Theoretical or empirical models are used in range-based localization techniques to translate RSS into estimates of distance. Range-based localization can achieve better accuracy but is costly in requiring either per-node ranging hardware [12] or careful system calibration and environment profiling [13], [14], and thus it is not appropriate for large-scale sensor networks. The correlation of noise due to shadowing from obstacles in wave propagation has been exploited to estimate the locations of transmitters [15]. Cumulative errors in measurement with positioning methods have been treated as problems with localization where data sampled over time have generated points in high dimensional space [16], [17], [18]. The multi-dimensional scaling (MDS) model has been used to reduce dimensionality to estimate locations [16]. However, the 
linear relationship requirement between correlation coefficients and radial distance in MDS has restricted its applications to wireless environments where RSS correlations are highly nonlinear if there is a radial distance [19] between receivers. Manifold learning (reduced nonlinear dimensionality) algorithms such as Isomap, Local Linear Embedding (LLE) and Hessian LLE have been used to centralize localization [17], [18]. The linearity between correlation measurements and radial distance is restricted in these approaches to a small area containing $\mathrm{K}$ nearest neighbors. However, the linearity between RSS and radial distance does not hold in Li and Liu [19], even in the immediate vicinity of operating frequencies greater than $10 \mathrm{MHz}$.

Range-free approaches localize nodes based on simple sensing, such as wireless connectivity [11], [20], [21] and anchor proximity [10], [22], [23]. Wireless connectivity information between neighboring nodes is used to estimate the location of a target node by using MDS [20]. Their major limitation is that they all rely on a large number of uniformlydistributed anchors in the networks. Embedding the combinatorial Delaunay complex in the landmark Voronoi diagram [21] has improved the localization of target nodes in various network topologies. However, using a number of landmarks to achieve precise accuracy in localization is costly.

The approximate-point-in-triangulation (APIT) algorithm [22] was proposed for area-based range-free localization, where all sensor nodes were localized by using the location information of GPS-equipped anchors. The areas occupied by sensor nodes were divided into many triangular regions between anchors in this approach by using the location information provided by GPS. This approach provided excellent accuracy when irregular radio patterns and random node placements were considered. Moreover, the large number of distributed anchors will counteract problems such as high deployment costs when applied to large areas. In Centroid [10], all possible anchors broadcast their location information to all other target nodes. The target nodes use the location information from anchors that are located in their vicinity to estimate their own location coordinates. The main difficulty with the centroid is the large number of anchors to be considered in the estimates. Moreover, if anchors are not uniformly distributed, the distance between them and target nodes varies, which deteriorates the accuracy of localization. It is necessary to take into consideration the distance between anchors and target nodes to solve this problem. The distances between anchors and target nodes are considered in the distance vectorhop (DV-hop) localization algorithm [11] and resilient Ethernet protocol (REP) [23] as a form of hop counting, which is a range-free approach that does not use RSS to compute the distance between nodes. DV-hop performs well when deployed sensor nodes have regular node density and distances between them. However, the resulting estimates may not be optimal if the radio patterns are irregular and random node deployment is used in practice.

In MoLPS, the coordinates of anchors were determined from the selected coordinates of footprints by using our proposed method. Instead of selecting all the anchors to estimate the location of target nodes, we select the anchors that had fewer variations in the radial distance which can minimize the uncertain radial distance contamination problem to the

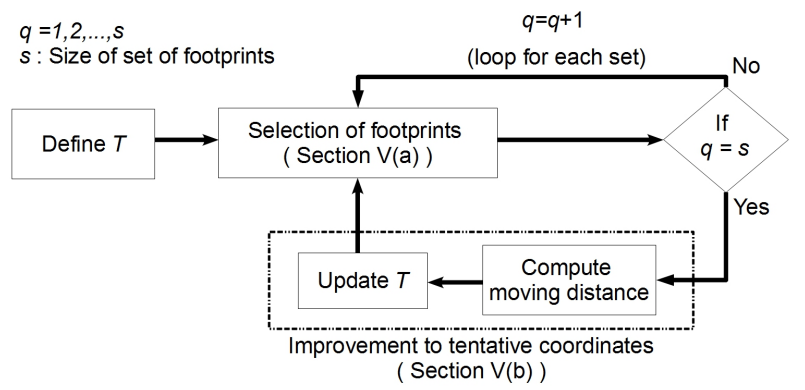

Fig. 2: Selection of footprints and improved $T$ in MoLPS.

localization of target nodes.

\section{Problem Definition}

We assumed that a mobile receiver would travel within a sensory boundary field that was deployed by sensor nodes that were transmitting their signals to the receiver on a periodic basis in this research. The mobile receiver is traveling at a constant speed while it is collecting signals from target nodes at $\tau$ intervals. The mobile receiver and target nodes are capable of communicating within their communication range $D$. Every time the mobile receiver receives a signal from target node $i$, it measures the RSS value of the signal and then stores it as tuple $\left(t, r_{i, t}\right)$, where $t$ is the time denoted as $t=t_{1}, t_{2}, \ldots, t_{\tau}$ and $r_{i, t_{j}}$ is an RSS value denoted as $r_{i, t_{j}}=r_{i, t_{1}}, r_{i, t_{2}}, \ldots, r_{i, t_{\tau}}$. Each tuple contains a different RSS value, each of which is collected from a different position of the footprint in each $t$.

We assumed that the ranging levels of RSS received from a target node would decrease due to path loss effects as the distances between each footprint and target node increased. All footprints $P_{k_{q}, q}=\left(X_{k_{q}, q}, Y_{k_{q}, q}\right)$ were divided into $s$ sets according to the path loss values. Here, $P_{k_{q}, q}$ denotes $k_{q}$-th footprints in set $q$ where $k_{q}=1,2, \ldots, m_{q}$ and $q=1,2, \ldots, s$ as listed in Table I.

We took into consideration noisy environments in measuring RSS that contributed to variations in radial distances between footprints that received the same RSS values from a target node. The average of the positions from surrounding footprints are used in the centroid to estimate the locations of target nodes [10]. Accuracy with this approach greatly depends on variations in the radial distances of a target node at each footprint. The propagation of wireless signals is ideal in a noise-free environment, such that a target node can communicate with a mobile receiver from any footprint that is located within a perfect sphere centered on the target node and with a radius equal to its standard interrogation range. It is possible in this case to estimate the position of a sensor node by averaging all footprint coordinates that are located within its radius. However, it is difficult to guarantee whether the radial distance of the target node will be accurate at each footprint in practice in noisy environments. Moreover, since the position of a true target node is unknown, there is no way of selecting footprints that have fewer variations in distance to estimate the position of a target node.

It is necessary to select footprints that have fewer variations in radial distance in range-free mobile localization that relies 


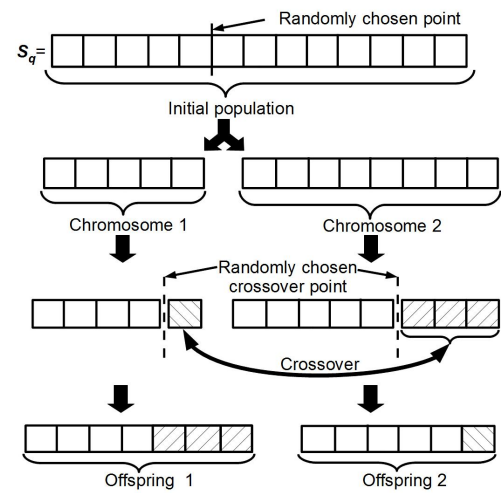

Fig. 3: The chromosome representation and generation process in GA.

on the average of the footprints as the positions of the estimates. For example, the mobile receiver in Fig.1(a) is traveling around the sensor node field and is collecting signals at each time interval and $d_{1}, d_{2}, \ldots, d_{6}$ denote the radial distances of a target node to all footprints. Assuming that there are two sets of footprint coordinates in Fig.1(b), localization using the information from $S_{e t}$ is more accurate than that from $\mathrm{Set}_{2}$. The small variations in radial distance between the target node and each footprint in $\mathrm{Set}_{1}$ contribute to greater accuracy. Therefore, it is important to select appropriate footprints that have fewer variations in radial distance to estimate the positions of target nodes and to obtain accurate localization.

\section{DESCRIPTION OF Algorithm}

We iteratively improved the position of tentative coordinates $T=\left(X^{T}, Y^{T}\right)$ in close proximity to the true position of a target node to estimate the position of the target node. Here, we used $T$ to select the collection of footprints that had a center coordinate nearest to $T$. The algorithm we propose is outlined in Fig. 2. First, we arbitrarily define $T$ in the sensory boundary field without any knowledge of the position of the target node. Then, we select the collection of footprints that have the nearest center coordinates to $T$ for each set $q$. After the footprints have been selected, we determine the moving distance for $T$ by evaluating the concentration of plotted center coordinates of selected footprints in the vicinity of $T$ by iteratively improving $T$ until the number of cycles of improvements to obtain the best solution is satisfied.

\section{A. Selection of footprints}

We selected collections of footprints that had fewer variations in radial distance to obtain accurate estimates of a target node with MoLPS. We used a genetic algorithm (GA) approach in this research to select $P_{k_{q}, q}$ by searching the nearest center coordinates of selected footprints to a tentative coordinate, $T$. The pseudocode for the GA in selecting the footprints is given in Algorithm 1. GA is a search algorithm that searches an optimal solution to solve a combinatorial problem, such as the NP-complete traveling salesman problem (TSP). The solution to a given problem is represented as a chromosome in GA. A population of solutions is created, and operators such as mutation and crossover are applied to derive the solutions.

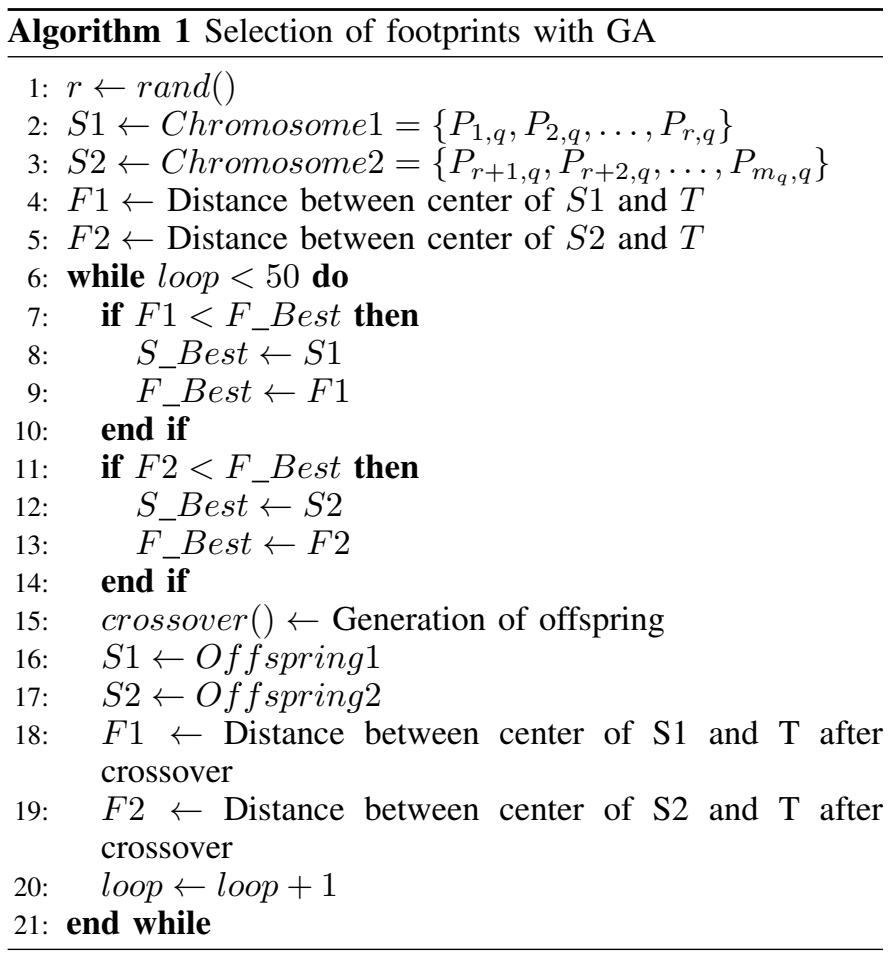

The relative accuracies (fitnesses) of the solutions are then compared to find the best solution.

Assuming we have a total of $m_{q}$ footprints in a $q$ set, we determine all footprints in $P_{k_{q}, q}$ as an initial solution, $S_{q}=$ $\left\{P_{1, q}, P_{2, q}, \ldots, P_{m_{q}, q}\right\}$. We determine the center coordinates of $S_{q}$ as $C_{q}$, which is computed as:

$$
C_{q}=\left(\frac{\sum_{k_{q}=1}^{m_{q}} X_{k_{q}, q}}{m_{q}}, \frac{\sum_{k_{q}=1}^{m_{q}} Y_{k_{q}, q}}{m_{q}}\right)
$$

The chromosome representation and the generation process in GA is outlined in Fig.3. We let $S_{q}$ represent the initial population in GA and compute the distance between $C_{q}$ and $T$ as the initial fitness of GA, $F_{q}$ as:

$$
F_{q}=\sqrt{\left(X^{T}-X^{C_{q}}\right)^{2}+\left(Y^{T}-Y^{C_{q}}\right)^{2}}
$$

where $\left(X^{C_{q}}, Y^{C_{q}}\right)$ denotes the center coordinate $C_{q}$ for $S_{q}$. We divide $S_{q}$ into two sets of footprints at randomly chosen points to represent two chromosomes in GA and compute the fitness of each chromosome as $F_{1, q}$ and $F_{2, q}$ using the same Eq. (2). The best solution is selected from a pair of chromosomes based on the least fitness (i.e., nearest distance) by comparing $F_{1, q}$ and $F_{2, q}$. A chromosome that has better fitness is selected as the best solution, $S_{q}^{b e s t}$.

Crossover is applied to generate offspring chromosomes from dominant parent chromosomes. The crossover operator separates each chromosome into two sets at randomly chosen crossover points and exchanges separate sets to form new offspring. If crossover does not occur, the new offspring are exact copies of their parent chromosomes. Then, GA is repeated using the new offspring until the number of iterations of the computation satisfies a bound (e.g., fifty).

$171 \mid \mathrm{P}$ a g e 
We assume the selected footprints at the last iteration of GA to be the best selection of footprints $S_{q}^{B e s t}=$ $\left\{P_{1, q}, P_{2, q} \ldots, P_{n_{q}, q}\right\}$ where $n_{q}$ is the total number of selected footprints. We search $S_{q}^{B e s t}$ for each set of footprints and use the center coordinates $C_{q}^{B e s t}$ of $S_{q}^{B e s t}$, which are computed with Eq. (3) to improve the position of $T$ as will be described in the next subsection.

$$
C_{q}^{\text {Best }}=\left(\frac{\sum_{k_{q}=1}^{n_{q}} X_{k_{q}, q}}{n_{q}}, \frac{\sum_{k_{q}=1}^{n_{q}} Y_{k_{q}, q}}{n_{q}}\right)
$$

\section{B. Improvements to tentative coordinate}

We compute a direction vector by evaluating the concentration of center coordinates $\left\{C_{1}^{\text {Best }}, C_{2}^{\text {Best }}, \ldots, C_{q}^{\text {Best }}, \ldots, C_{s}^{B e s t}\right\}$ in the vicinity of $T$ to improve the position of $T$.

As can be seen from Fig. 5, we assume a square region centered at $T$ that is divided into $3 \times 3$ frames. The square region has an $A$ length along each side that initially covers all the center coordinates. We call the square region the sequence spatial density (SSD). Each frame contains a value that indicates the number of $C_{q}^{B e s t}$ coordinates.

Let $I_{x, y}$ denote the number of $C_{q}^{B e s t}$ points in the frame $(x, y)$ where $x, y$ are the indexes of the frames in SSD shown in Fig. 5. We determine a direction vector by computing the partial derivatives of SSD as the sum of the differences between two adjacent frames in SSD as:

$$
\begin{aligned}
\frac{\partial I}{\partial x}= & \left(I_{2,1}-I_{1,1}\right)+\left(I_{3,1}-I_{2,1}\right) \\
& +\left(I_{2,2}-I_{1,2}\right)+\left(I_{3,2}-I_{2,2}\right) \\
& +\left(I_{2,3}-I_{1,3}\right)+\left(I_{3,3}-I_{2,3}\right) \\
\frac{\partial I}{\partial y}= & \left(I_{1,2}-I_{1,1}\right)+\left(I_{1,3}-I_{1,2}\right) \\
& +\left(I_{2,2}-I_{2,1}\right)+\left(I_{2,3}-I_{2,2}\right) \\
& +\left(I_{3,2}-I_{3,1}\right)+\left(I_{3,3}-I_{3,2}\right)
\end{aligned}
$$

We compute the partial derivatives of SSD horizontally $\frac{\partial I}{\partial x}$ and vertically $\frac{\partial I}{\partial y}$ to compute the direction vector at each $h$ th cycle of the improvements by using the direction vector function, $\overrightarrow{D V F}$ as:

$$
\overrightarrow{D V F}_{h}=\left(\frac{\partial I}{\partial x}, \frac{\partial I}{\partial y}\right)
$$

We improve $T_{h}$ by using $\overrightarrow{D V F}_{h}$ with length $\Delta T_{h}$ proportional to the vector's magnitude, $\left|\overrightarrow{D V F}_{h}\right|$, computed as:

$$
\begin{gathered}
\left|\overrightarrow{D V F}_{h}\right|=\sqrt{\left(\frac{\partial I}{\partial x}\right)^{2}+\left(\frac{\partial I}{\partial y}\right)^{2}} \\
\Delta T_{h}=\left(\frac{\frac{\partial I}{\partial x}}{\left|\overrightarrow{D V F}_{h}\right|} \times \nu, \frac{\frac{\partial I}{\partial y}}{\left|\overrightarrow{D V F}_{h}\right|} \times \nu\right)
\end{gathered}
$$

Here, $\nu$ denotes a scale factor parameter for the unit vector computed from $\overrightarrow{D V F}_{h}$ to determine the length of improvement, $\Delta T_{h}$. We improve the position of $T_{h}$ with direction vector $\Delta T_{h}$ for the next cycle of improvement as:

$$
T_{h+1}=T_{h}+\Delta T_{h}
$$

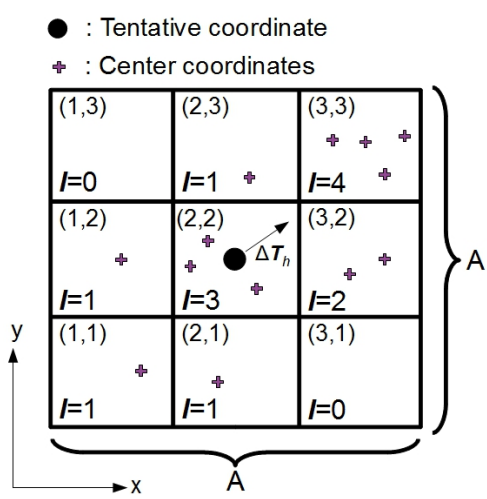

Fig. 5: Frames of Sequence of Spatial Density (SSD)

The improvement of $T$ will also affect the concentration of center coordinates. As we can see from Fig. 4, $T$ has improved its position approaching the true positions of target nodes at 20, 40, and 60 cycles. The concentration of center coordinates has also simultaneously improved as they are computed by the average of selected footprints that are nearest to $T$ as described in Subsection V-A.

Therefore, instead of using the same values of parameters $A$ and $\nu$ in all cycles, we reduced both parameters by the fraction of $a / b$ every $m$ cycles of improvement to avoid phenomena where improvement was not taking effect because the frames were too large, as shown in Fig. 7. We called these phenomena zero vector effects, where the direction vector became zero as all the center coordinates were located inside the center frame of SSD. If none of the center coordinates are located in the frame other than the center frame of SSD, the direction vector will become zero as they are computed from the sum of the differences between two adjacent frames.

\section{PERFormance EVALUATION}

We conducted a simulation on the proposed algorithm to evaluate the performance of MoLPS to localize sensor nodes in a noisy environment. This simulation experiment was used to demonstrate what effect a noisy environment and the shrinking size of SSD had on the localization error of sensor nodes.

The remaining part of this section presents the simulation setup, path loss model, and the results we obtained from evaluating performance.

\section{A. Simulation Setup}

We implemented the algorithm in a custom $\mathrm{C}$ simulator, where we randomly deployed a set of 100 sensor nodes with one mobile receiver traveling in a $50 \mathrm{~m} \times 50 \mathrm{~m}$ square region at a constant speed, as seen in Fig. 6. The mobile receiver and sensor nodes had the same communication range of $10 \mathrm{~m}$.

The mobile receiver traveled in a sensory boundary field and received signals from sensor nodes within their communication range at each time interval $t$. The positions of sensor nodes were estimated with our proposed algorithm by measuring the direction vector of the SSD from $T$. The tentative coordinate was arbitrarily deployed within the sensory 


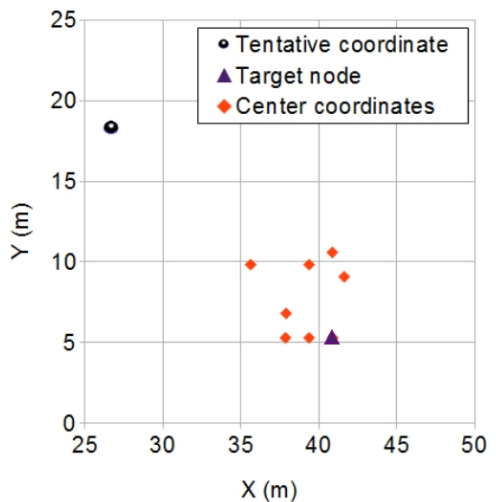

(a) Number of cycles $=20$

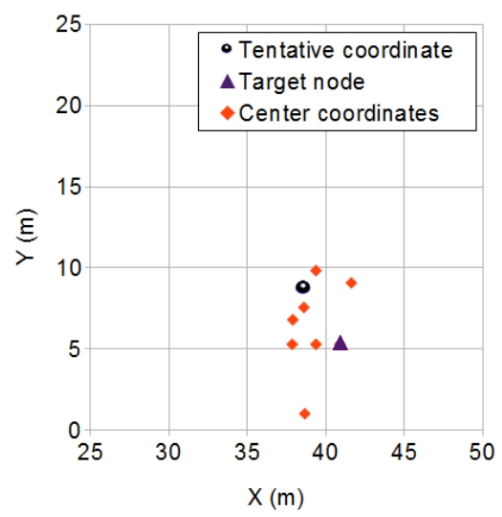

(b) Number of cycles $=40$

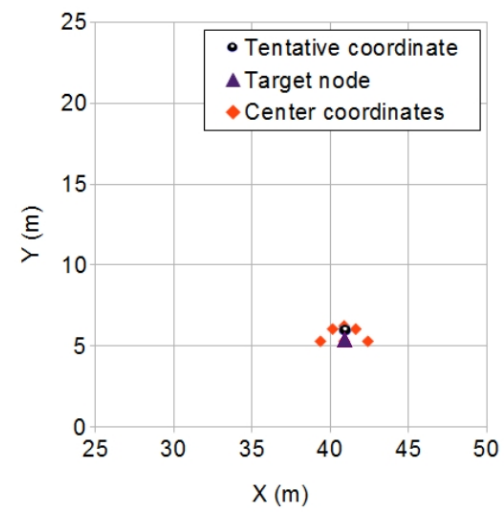

(c) Number of cycles $=60$

Fig. 4: Improvement of $T$ approaching true position of target node

boundary field. We deployed the tentative coordinate in the first cycle of improvement at the center of the sensory boundary field as an initial coordinate of $T$. The SSD frames were used to compute the moving distance of $T$ in which the initial value of $\nu$ was $70 \mathrm{~m}$ per number of cycles and the initial value of $A$ was $50 \mathrm{~m}$ where all center coordinates were included in the coverage area of SSD for each tentative coordinate.

We defined the criterion in MoLPS for the error in localization as the difference between $T$ and the true position of a target node in the cycle of improvement, $h$. Localization error indicated the degree of accuracy in estimates that the algorithm could achieve.

\section{B. Path Loss model}

We used an extended model of log-distance path loss by combining it with the DoI model [22]. The log-distance path loss model is used in many indoor and outdoor environments in which multipath propagation is presented.

The RSS reading was a value from our degree of irregularities (DoI) extended log-distance path loss in Eq. 9. There is a plot of the path loss values with our model in Fig. 8.

$$
P L=\left\{\left(P L_{o}+10 \gamma \log \frac{d}{d_{o}}\right) \times(1 \pm(\operatorname{rand}() \times D o I))\right\}+S
$$

Here, $d_{o}$ is the reference distance (i.e., $1 \mathrm{~m}$ ) and $P L_{o}$ denotes the path loss in decibels at $d_{o}$, which was assumed to be $47 \mathrm{~dB}$. The $d$ is the distance between sensor nodes and the mobile receiver computed from the real coordinates of the simulation system. The $\gamma$ refers to the path loss exponent, which depends on channels and the environment. According to residential indoor models [24], the path loss exponent, $\gamma$, in this model is a random variable, and requires sufficient measurements on the spot in various residential environments before effectively being applied to generic scenarios. We have used the measurements in Sohrabi et al. [25] in this paper, which denote the value of average path-loss exponents as 1.9 in an engineering building. The $S$ is log-normal shadow fading in decibels. The $S$ is usually a random variable with a Gaussian distribution with zero mean and standard deviation $\sigma$, which was assumed to be 5.7 according to Sohrabi et al. [25]. The

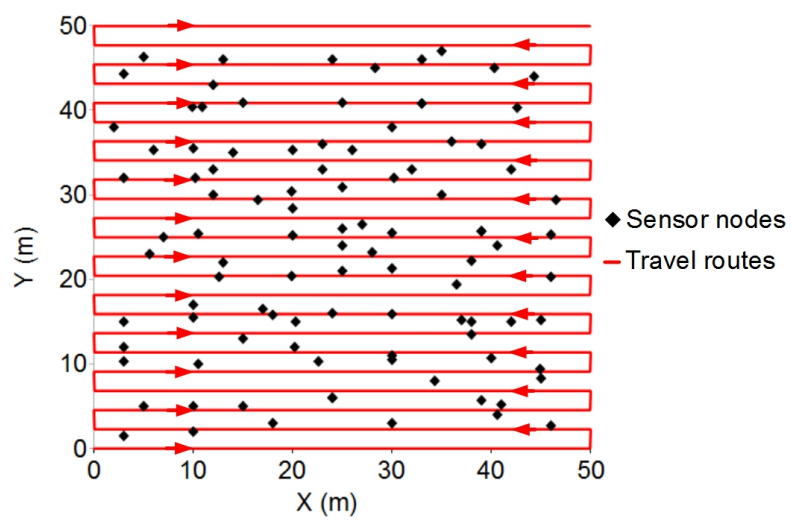

Fig. 6: Deployment of sensor nodes.

DoI is the radio irregularity and $\operatorname{rand}()$ is a random number, $\mathcal{U}(0,1)$. We ran five simulations with different $D o I$ values in a range of $0 \sim 1.0$.

\section{Experiment Results}

We compared the cumulative distribution function (CDF) of cycles for the number of nodes that had their localization error reduced below $2 \mathrm{~m}$ with different values of parameters $a$ and $b$ when parameters $A$ and $\nu$ were reduced by the fraction of $a / b$ through cycles of improvement. As shown in Fig. 9, less than $71 \%$ of tentative coordinates had their localization error reduced below $2 \mathrm{~m}$ approaching the true position of target nodes when the number of cycles reached 35 where parameters $A$ and $\nu$ were reduced by the fraction of $a / b=1 / 4$. However, the percentages were larger where parameters $A$ and $\nu$ were reduced by the fraction of $a / b=3 / 4$, as seen in Fig. 10. In this case, the percentage of tentative coordinates that had their localization error reduced below $2 \mathrm{~m}$ was more than $80 \%$ when the number of cycles reached 80 .

The size of the square region for sequence spatial density (SSD) has an impact on computing the direction vector 


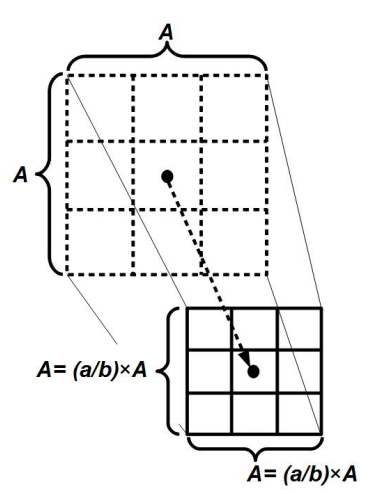

Fig. 7: Reduced $A$ length for SSD every $m$ cycles of improvement.

to improve $T$. A larger SSD will increase the number of center coordinates included in SSD that increases the total value of differences between the number of center coordinates between two adjacent frames. However, if $A$ is reduced too much between the two cycles of improvement, the number of center coordinates that are included in SSD will decrease. The decreased number of center coordinates in SSD will increase the possibility of zero vector effects that take place when the center coordinates are only located in the center frame of SSD. As we can see from Fig. 9, the zero vector effects took place when the number of cycles reached 35 and many of the center coordinates were excluded from SSD because the value of parameter $A$ was reduced too much when $a / b=1 / 4$ compared to the improvement in Fig. 10 where the $T$ coordinates were continuously improved when $a / b=3 / 4$ until the last number of cycles.

We also compared the required number of cycles to improve the tentative coordinates in different numbers of footprints. We fixed two values of localization error as a threshold in this evaluation scenario to assess how many cycles were needed for the tentative coordinates to improve their positions below these two thresholds (i.e., $2 \mathrm{~m}$ and $5 \mathrm{~m}$ ). The average number of cycles for each tentative coordinate was used to represent how many cycles were required for each number of footprints. As shown in Figs. 11 and 12, the numbers of cycles were almost equal in all numbers of footprints under both conditions. However, the parameter of $a / b$ affected the number of cycles that reduced the localization error of $T$. The tentative coordinates required less than 21 cycles $(2 \mathrm{~m})$ and 17 cycles $(5 \mathrm{~m})$ for each threshold in which parameters $A$ and $\nu$ were reduced by the fraction of $a / b=1 / 4$, as seen in Fig. 11 . However, the tentative coordinates required greater numbers of cycles to reduce their localization error below $2 \mathrm{~m}$ and $5 \mathrm{~m}$, as seen in Fig. 12. They needed 46 cycles for the former and 35 cycles averagely for the latter in which parameters $A$ and $\nu$ were reduced by the fraction of $a / b=3 / 4$.

SSD reduced by a large fraction of $a / b$ yielded a small difference in the number of center coordinates in the frames in SSD between cycles compared to the condition in which SSD was reduced by a small fraction of $a / b$. The small fraction of $a / b$ enabled SSD to reduce its size by a larger $A$, which created large differences in the number of center coordinates in each frame as the center coordinates that were located were

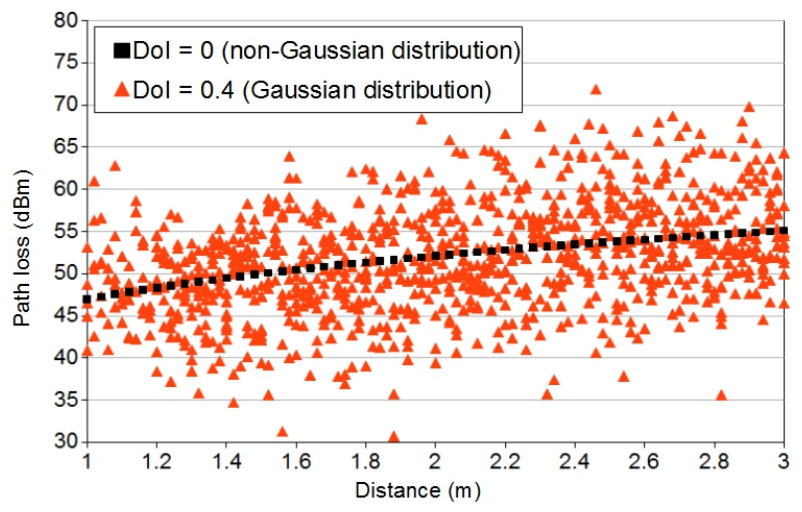

Fig. 8: Plot of path loss values

separated from one another. These will increase the value of the moving distance that improved the position of $T$ in fewer numbers of cycles.

We also compared what impact DoI had on localization error in the estimates of target node positions in various locations. The average localization error for our proposed algorithm was not entirely different for all DoI values, as seen in Fig. 13. MoLPS use range-free approaches that only use RSS to detect the proximity of footprints. Therefore, the irregularities in RSS did not have a huge impact on localization error in any target nodes on average as they did not directly use the RSS values as a metric to estimate the position of target nodes. The mean number of cycles under both conditions where the threshold of localization error was set to $2 \mathrm{~m}$ and $5 \mathrm{~m}$ corresponded to about 45 and 34 .

\section{CONCLUSION}

We proposed range-free mobile localization based on the proximities of selected footprints in noisy environments. We used GA to iteratively search the best selection of footprints that had the nearest center coordinates to $T$. We improved the positions of tentative coordinates by measuring the direction vector from the concentration of center coordinates in the vicinity of $T$. The footprints in our proposed algorithm were divided into sets by using ranging levels to decrease variations in the radial distance between footprints in a set. We evaluated our method based on a variety of metrics that proved that it was resistant to the number of footprints used in calculations and high DoI environments at a given number of cycles while providing low localization error.

The ability to localize sensor nodes without any reference nodes in noisy environments for mobile localization can improve the localization environment in large areas. However, determining the estimates of target node positions still remains unsolved as we determined the positions of estimates by continuously improving the tentative coordinates approaching the true target nodes until the number of cycles to improve tentative coordinates satisfied a boundary (i.e., 100 cycles).

Determining suitable values for parameters $A$ and $\nu$ were major causes of difficulties in our investigations. We plan to 


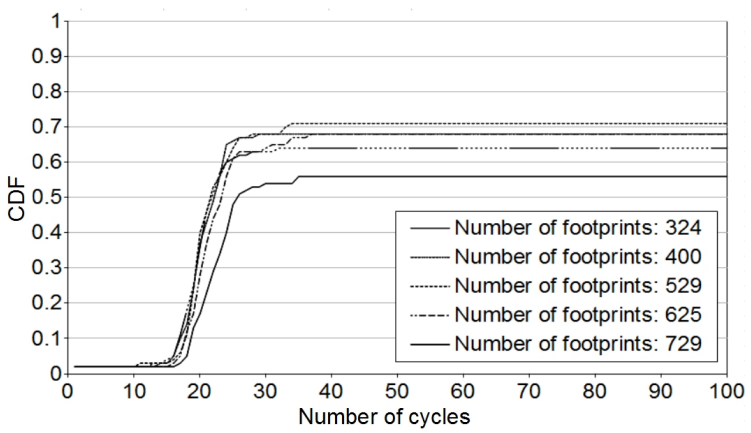

Fig. 9: Cumulative distribution of number of cycles for number of nodes that had their localization error reduced below $2 m$ when parameters $A$ and $\nu$ were reduced by fraction of $a / b=$ $1 / 4$.

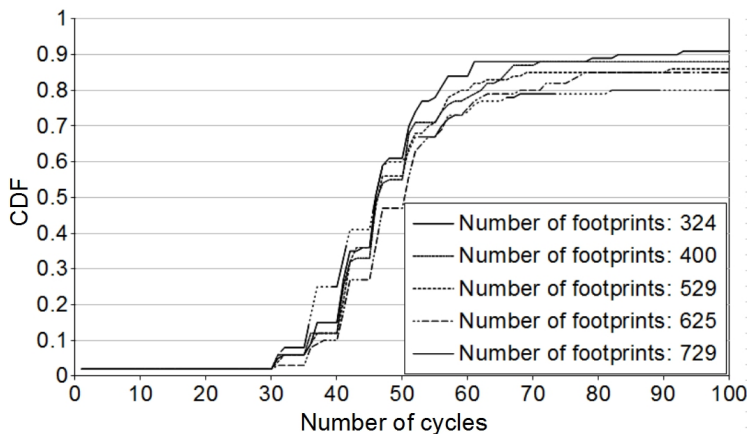

Fig. 10: Cumulative distribution of number of cycles for number of nodes that had localization error reduced below $2 m$ when parameters $A$ and $\nu$ were reduced by fraction of $a / b=3 / 4$.

design a method of determining receiver mobility to obtain accurate estimates by using localization based on proximity techniques. We also plan to apply our method to a real environment by running empirical experiments that focus on accurate proximity-based estimates of positions for mobile localization in the future.

\section{REFERENCES}

[1] F. Akyildiz, W. Su, Y. Sandarasurbramaniam, and E. Cayirci, "Wireless sensor networks: A survey," Computer Networks Journal, vol. 38, no. 4, pp. 393-422, 2002.

[2] M. Ang, Y. F. Lim, and M. Sim, "Robust storage assigment in unitload warehouses," Journal Management Science, vol. 58, no. 11, pp. 2114-2130, 2012.

[3] J. Maneesilp, C. Wang, H. Wu, and N. F. Tzeng, "RFID Support for Accurate 3-Dimensional Localization," in IEEE Transactions on Computers, 2012.

[4] G. Zhou, T. He, S. Krishnamurthy, and J. A. Stankovic, "Models and solution for radio irregularity in wireless sensor networks," ACM Trans. on Sensor Networks, vol. 2, no. 2, pp. 221-262, 2006.

[5] K. Fukuda, and E. Okamoto, "Performance improvement of TOA Localization Using IMR-Based NLOS Detection in Sensor Networks," in International Conference on Information Networking, 2012, pp. 1318.

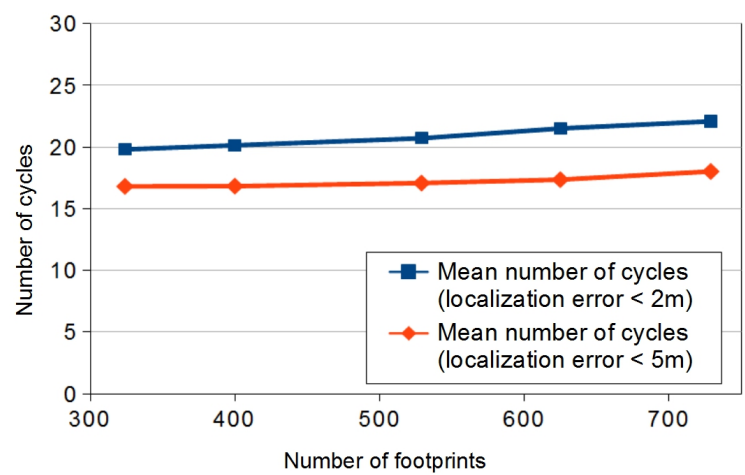

Fig. 11: Effect of localization error on number of cycles when parameters $A$ and $\nu$ are reduced by fraction of $a / b=1 / 4$.

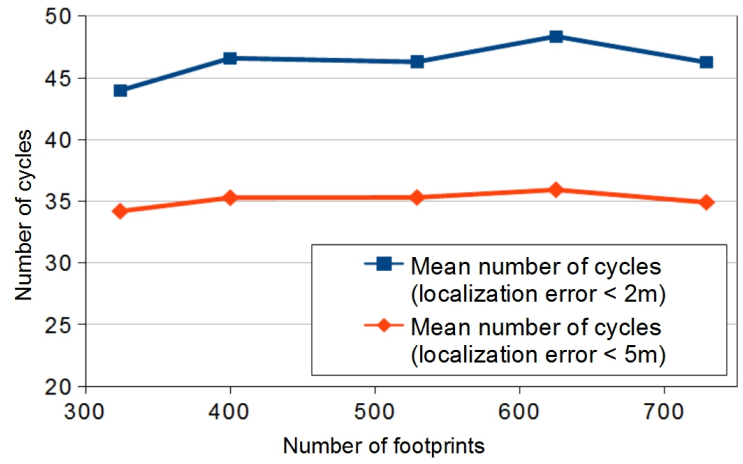

Fig. 12: Effect of localization error on number of cycles when parameters $A$ and $\nu$ are reduced by fraction of $a / b=3 / 4$.

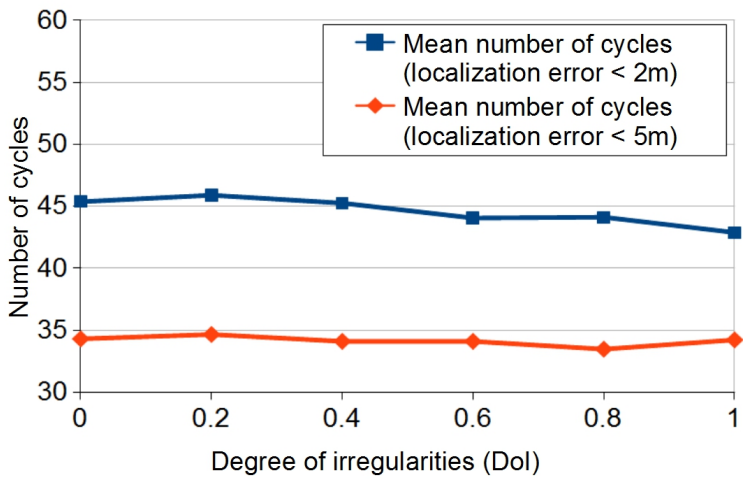

Fig. 13: Effect of DoI on number of cycles.

[6] B. Xu, Guodong, R. Yu, and Z. Yang, "High-accuracy tdoa-based localization without time synchronization," IEEE Trans. on Parallel and Distribution Systems, 2012.

[7] D. Niculescu, and B. Nath, "Ad Hoc Positioning System (APS) Using AOA," in 22nd Annual Joint Conference of the IEEE Computer and Communications. IEEE Societies, 2003, pp. 1734-1743.

[8] K.Whitehouse, C. Karlof, and D. Culler, "A practical evaluation of radio signal strength for ranging-based localization," ACM SIGMOBILE Mobile Computing and Communications Review, vol. 11, no. 1, pp. 4152, 2007. 
[9] K. Srinivasan, P. Dutta, A. Tavakoli, and P. Levis, "Understanding the causes of packet delivery success and failure in dense wireless sensor networks," in Proc. of the 4th International Conference on Embedded Networked Sensor Systems, 2006, pp. 419-420.

[10] N. Bulusu, J. Heidemann, and D. Estrin, "Gps-less low cost outdoor localization for very small devices," IEEE Personal Communication Magazine, vol. 7, no. 5, pp. 28-34, 2000.

[11] D. Niculescu, and B.Nath, "Dv based positioning in ad hoc networks," Journal of Telecommunication Systems, vol. 22, no. 1-4, pp. 267-280, 2003.

[12] N. B. Priyantha, A. Chakraborty, and H. Balakrishnan, "The Cricket location-support system," in Proc. of the 6th Annual International Conference on Mobile Computing and Networking, 2000, pp. 32-43.

[13] P. Bahl, and N. Padmanabhan, "RADAR: An In-Building RF-based User Location and Tracking System," in 19th Annual Joint Conference of the IEEE Computer and Communications. IEEE Societies, 2000, pp. 775-784.

[14] A. Savvides, C. C. Han and M. B. Strivastava, "Dynamic Fine-grained Localization in Ad-hoc Networks of Sensors," in Proc. of the 7th annual international conference on Mobile computing and networking, 2001, pp. 166-179.

[15] P. Agrawal, and N. Patwari, "Correlated link shadow fading in multi-hop wireless networks," IEEE Transactions on Wireless Communications, vol. 8, no. 8, pp. 4024-4036, 2009.

[16] J. Xiang, and Z. Hongyuan, "Sensor positioning in wireless ad-hoc sensor networks using multidimensional scaling," in 23rd Annual Joint Conference of the IEEE Computer and Communications. IEEE Societies, 2004, pp. 2652-2661.

[17] N. Patwari, and A. O. Hero, "Manifold learning algorithms for localization in wireless sensor networks," in IEEE International Conference on Acoustics, Speech, and Signal Processing, 2004. Proceedings. (ICASSP '04), 2004, pp. 857-860.

[18] W. Chengqun, C. Jiming, S. Youxian, and S. Xuemin, "Wireless Sensor Networks Localization with Isomap," in IEEE International Conference on Communications, 2009. ICC '09, 2009, pp. 1-5.

[19] M. R. Basheer, and S. Jagannathan, "Localization of objects using stochastic tunneling," in IEEE Wireless Communications and Networking Conference (WCNC), 2011, pp. 587-592.

[20] Y. Shang, W. Ruml, Y. Zhang, and M. P. J. Fromherz, "Localization from mere connectivity," in Proc. of the 4th ACM international symposium on Mobile ad hoc networking and computing, 2003, pp. 201-212.

[21] S. Lederer, Y. Wang. and J. Gao, "Connectivity-based localization of large-scale sensor networks with complex shape," ACM Transactions on Sensor Networks (TOSN), vol. 5, no. 4, 2009.

[22] T. He, C. Huang, B. M. Blum, J. A. Stankovic, and T. Abdelzaher, "Range-Free Localization Schemes for Large Scale Sensor Networks," in Proc. of MobiCom, 2003, pp. 81-95.

[23] M. Li, and Y. Liu., "Rendered Path: Range-Free Localization in Anistropic Sensor Networks With Holes," in Proc. of MobiCom, 2007, pp. 51-62.

[24] S. S. Ghassemzadeh, R. Jana, C. W. Rice, W. Turin, V. Tarokh, "Measurement and modeling of an ultra-wide bandwidth indoor channel," Transactions of Communication, vol. 52, no. 10, pp. 1786-1796, 2004.

[25] K. Sohrabi, B. Manriquez, and G. Z. Pottie, "Near Ground Wideband Channel Measurement in 800-1000MHz," in Proc. of IEEE Vehicular Technology Conference, 1999, pp. 1222-1226. 